\title{
Rezension: Sandra Brändli, Die Flexibilität urheberrechtlicher Schrankensysteme
}

\begin{abstract}
Alfred Früh *
Wie könnte das urheberrechtliche Schrankensystem der Schweiz mit mehr Flexibilität versehen werden, um besser auf technologische Neuerungen reagieren zu können? Die Autorin untersucht diese Forschungsfrage in ihrer ambitionierten Dissertation mit einem rechtsvergleichenden Ansatz. Gerade der Blick auf die sehr flexible US-amerikanische «fair use»-Schranke verspricht eine Inspiration für die Schweiz und führt zur Frage, ob auch hierzulande eine General- oder Öffnungsklausel den Schrankenkatalog erweitern sollte. Die gründliche Untersuchung führt aber zu erstaunlichen Einsichten: Selbst wenn gute Gründe für eine General- oder Öffnungsklausel sprechen, besteht bereits heute eine nicht unerhebliche Flexibilität bei der Anwendung der Schranken. Zudem können auftretende Probleme oft besser auf der Ebene des Ausschliesslichkeitsrechts gelöst werden als aufjener der Schranken.
\end{abstract}

Zitiervorschlag: Alfred Früh, Rezension: Sandra Brändli, Die Flexibilität urheberrechtlicher Schrankensysteme, in: sui-generis 2018, S. 200

URL: sui-generis.ch/68

DOI: $\quad$ https://doi.org/10.21257/sg.68

* Dr. Alfred Früh (alfred.frueh[at]rwi.uzh.ch), RA, ist Postdoc an der rechtswissenschaftlichen Fakultät der Universität Zürich und Geschäftsführer des Center for Information Technology, Society, and Law (ITSL).

Dieses Werk ist lizenziert unter einer Creative Commons Namensnennung - Weitergabe unter gleichen Bedingungen 4.0 International Lizenz. 
Mehr noch als andere Rechtsgebiete wird das Urheberrecht von praktisch jeder technischen Entwicklung neu herausgefordert. Anpassungsbedarf besteht insbesondere im Bereich der urheberrechtlichen Schranken - also bei jenen Einschränkungen des Urheberrechts zulasten des Rechteinhabers, welche Dritt- oder Allgemeininteressen verwirklichen sollen. Mit jedem technischen Entwicklungsschub sind neue Schranken ins Urheberrecht aufgenommen worden. Ohne diese Anpassungen wären beispielsweise heute die Dekompilierung von Computerprogrammen oder das Surfen im Internet (aufgrund der flüchtigen Speicherung von urheberrechtlich geschützten Werken im Cache des Computers) immer noch dem Urheber vorbehaltene Handlungen.

2 Die Gelegenheiten zum Nachvollzug der technischen Entwicklungen durch den Gesetzgeber sind rar: Zwischen den Revisionen des Urheberrechts verstreichen in der Schweiz im Schnitt etwa 30 Jahre genügend Zeit also, um die eine oder andere Rechtsunsicherheit entstehen $\mathrm{zu}$ lassen.

Sandra Brändli legt ihrer St. Galler Dissertation die Prämisse zugrunde, dass es auch anders ginge. Dass sie damit richtig liegt, zeigt bereits ein flüchtiger Blick in ausländische Rechtsordnungen. Gerade die angelsächsischen Copyright-Systeme scheinen im Vergleich mit den kontinentaleuropäischen Droit d'auteur-Gesetzen mehr Raum zu bieten, um technische Entwicklungen zu berücksichtigen. Dies führt zur Forschungsfrage, ob und wie das urheberrechtliche Schrankensystem in der Schweiz mit mehr Flexibilität versehen werden könnte. Die Autorin wählt dazu einen rechtsvergleichenden Ansatz und untersucht neben dem Schweizer Recht auch die Rechtsordnungen von Deutschland, der Europäischen Union, des Vereinigten Königreichs und der Vereinigten Staaten. Brändli verfolgt diesen breiten Ansatz mit grosser Konsequenz. Dabei beeindruckt die Gründlichkeit, mit der sie Auslegungsspielräume untersucht. Ebendiese Gründlichkeit führt aber auch zu ein paar Dopplungen, was indes bei einem Werk dieses Umfangs nicht weiter erstaunlich ist.

4 Der Zeitpunkt, in dem das Buch erscheint, ist günstig: Zum einen hat soeben die Konfrontation mit der nächsten «Generation» technischer Fragestellungen wie dem text and data mining begonnen ${ }^{1}$, zum anderen steht wieder einmal - bzw. immer noch die Revision des Schweizer URG vor der Tür. Im Dezember 2017 hat der Bundesrat einen Entwurf präsentiert. Die Rechtskommission des Nationalrats hat bereits Anhörungen dazu abgehalten und wird die Eintretensdebatte und die Detailberatung im dritten Quartal 2018 aufnehmen².

5 Die Arbeit ist in fünf Teile gegliedert. Sie beginnt mit einem Grundlagenteil (I.), woraufhin für alle Rechtsordnungen die Wesensmerkmale ihrer Schrankenordnung herausgearbeitet werden (II.). Anschliessend wird anhand konkreter Beispiele der Umgang der Schrankensysteme mit technischen Herausforderungen dargestellt (III.). Auf dieser Basis wird die Flexibilität der Schrankensysteme in den einzelnen Rechtsordnungen bewertet

1 Siehe dazu Melanie Graf/Kirsten Johanna Schmidt, Data Mining und wissenschaftliche Forschung - de lege lata und de lege ferenda, in: suigeneris 2017, S. 185.

2 Vgl. Medienmitteilung der RK-N vom 18. Mai 2018, Revision des Urheberrechts: Kommission beginnt Beratung mit Anhörungen. 
(IV.). Die Arbeit schliesst mit einer Diskussion möglicher Lösungen für das Schweizer Recht (V.).

Im Grundlagenteil (I.) zeigt sich schon bei der Erfassung des Untersuchungsgegenstands «Schranke», wie anspruchsvoll das Thema ist: Der Begriff der Schranke ist zwar im deutschsprachigen Raum etabliert, wird aber im Detail sehr unterschiedlich aufgefasst. Im angelsächsischen Raum werden demgegenüber die Begriffe der limitations und exceptions verwendet, wobei auch diese keineswegs überall gleich aufgefasst werden und wohl ohnehin nur im Kontext der jeweiligen Rechtsordnung zu verstehen sind. Vollständige Klarheit schafft die Arbeit hier nicht. Das ist aber - wie sich später zeigt - kein Mangel; auch ohne diese Klarstellungen lassen sich die Normen der einzelnen Rechtsordnungen miteinander vergleichen.

7 Interessant sind die Ausführungen zur Rechtsnatur der Schranken, etwa dazu, ob die Schranken dispositiv oder zwingend sind und ob sie gegenüber technischen Schutzmassnahmen durchsetzbar sind. Der Vergleich der Rechtsordnungen ergibt in Bezug auf beide Fragen ein gemischtes und mit einigen Rechtsunsicherheiten gespicktes Bild. Schrankensysteme existieren gemäss Brändli in drei unterschiedlichen Ausprägungen: (1) Als Liste von ausführlich ausformulierten Urheberrechtsschranken in den Droit d'auteur-Staaten, (2) als Schrankenkataloge, kombiniert mit fair dealingSchranken in Commonwealth-Staaten und (3) in Form einer fair use-Schranke, kombiniert mit einer Aufzählung detaillierter Urheberrechtsschranken in den USA. Deutliche Unterschiede gibt es auch auf der Rechtsfolgeseite. In Droit d'auteur-Staaten sind Schranken meist vergütungspflichtig, in den Commonwealth -Staaten und den USA meist vergütungsfrei.

8 Im Grundlagenteil skizziert die Autorin überdies die Instrumente, aus denen sich flexible rechtliche Systeme konstruieren lassen. Es sind dies Generalklauseln, Normen mit zeitlicher Verfallsfrist, finale Rechtssätze und das Operieren mit Prinzipien. Bei der Frage, wie diese Instrumente verbunden werden sollen, lässt die Autorin eine Präferenz für ein bewegliches System erkennen - einen Mittelweg zwischen starren Tatbestandsmerkmalen und offenen Generalklauseln, bei dem die einzelnen Elemente blosse Faktoren sind, nicht aber kumulativ notwendige oder alternativ bzw. einzeln hinreichende Bedingungen.

9 Was im Grundlagenteil fehlt, ist eine vertiefte Auseinandersetzung mit dem Sinn und Zweck der Flexibilität, die letztlich zur Frage führen würde, weshalb die neuen urheberrechtlich relevanten Nutzungshandlungen überhaupt frei bleiben sollen oder aber von Schranken erfasst sein müssen. Das Entwickeln eines analytischen Rahmens - über die (vorhandenen) grundsätzlichen Ausführungen zur ökonomischen Funktion der Schrankenbestimmungen hinaus - hätte den Rahmen der Arbeit aber wohl gesprengt.

10 Was die konkreten Ergebnisse betrifft, verblüfft mit Blick auf das Schweizer Urheberrecht zunächst einmal die Tatsache, dass die Aufzählung der Ausschliesslichkeitsrechte nur exemplarisch und nicht abschliessend zu verstehen ist, in Bezug auf den abschliessenden Charakter des 
Schrankenkatalogs aber keine Klarheit herrscht. Anders als in Deutschland, wo der Schrankenkatalog abschliessend zu verstehen ist, legt Brändli für die Schweiz dar, dass Analogieschlüsse zu bestehenden Schranken nicht a priori ausgeschlossen sind. Gerade aus dem Zweck des Interessenausgleichs ergebe sich, dass Lücken mit Analogieschlüssen geschlossen werden können sollen, was zum Ergebnis führt, dass der Schrankenkatalog nicht abschliessend ist. Dies ebnet den Weg für ungeschriebene, d.h. richterrechtlich veranlasste und nicht kodifizierte Schranken. Diese Flexibilität hat aber Grenzen. Zulässig ist gemäss Brändli nur die Einzelanalogie und die teleologische Extension (von Schranken) oder Reduktion (von Ausschliesslichkeitsrechten), nicht aber eine Gesamtanalogie.

Mit dieser Erkenntnis ist der Spielraum im Schweizer Recht bereits grösser als im EU-Ausland. Zwar sind auch dort flexible Lösungen nicht grundsätzlich ausgeschlossen. Aber zum einen steht der vom EuGH immer wieder erwähnte Grundsatz, Schranken seien eng auszulegen, solchen Lösungen entgegen. Zum anderen schränkt Art. 5 Abs. 5 der InfosocRichtlinie3 mögliche Flexibilisierungen durch den dort explizit erwähnten Dreistufentest grundlegend ein, was zumindest eine «ausgewogene Interpretation»4 des Dreistufentests voraussetzt, um doch

3 Richtlinie 2001/29/EG des Europäischen Parlaments und des Rates vom 22. Mai 2001 zur Harmonisierung bestimmter Aspekte des Urheberrechts und der verwandten Schutzrechte in der Informationsgesellschaft vom 22. Mai 2001.

4 S. hierzu Christophe Geiger/Reto M. Hilty/ Jonathan Griffiths/Uma Suthersanen, Declaration - A Balanced Interpretation of the «ThreeStep Test» in Copyright Law, JIPITEC 2010, $119 \mathrm{ff}$. noch zu einer anpassungsfähigen Schrankensystematik zu gelangen. Das Vereinigte Königreich leistet sich zwar eine aus der Rechtsprechung abgeleitete Schranke im öffentlichen Interesse5. Solange das EU-Recht für das Vereinigte Königreich noch gilt, stellt sich aber die Frage, ob diese Schranke im Lichte der Infosoc-Richtlinie überhaupt zulässig ist.

12 Andererseits reicht der Spielraum des Schweizer Rechts weniger weit als in den USA, wo die durch die Rechtsprechung entwickelte und lange nicht kodifizierte fair use-Schranke eine gewisse Offenheit erlaubt. Die fair use-Schranke beruht auf vier Fairness-Faktoren, welche in einer Gesamtbeurteilung gewichtet werden und entspricht damit dem angestrebten (oben in Rn. 8 erwähnten) beweglichen System. Der Nutzen der fair useSchranke hängt jedoch davon ab, ob ihr die (höchstrichterliche) Rechtsprechung klare Konturen geben kann. Dazu fehlen aber leider weitere Ausführungen - genauso wie zur Frage, wie in den USA mit den internationalen Vorgaben des Dreistufentests umgegangen wird.

13 Bei der Analyse konkreter Beispiele (III.) wird sodann deutlich, dass durch technische Entwicklungen entstehende Probleme beim urheberrechtlichen Interessenausgleich auf ganz verschiedenen Ebenen gelöst werden können: Neben der Anpassung der Schranken kann nämlich auch die Reichweite des Ausschliesslichkeitsrechts begrenzt werden. Brändli verweist hier zu Recht exemplarisch auf den Pressespiegel-Entscheid des Bundesgerichts,

5 S. William Cornish/David Llewelyn/Tanya Alpin, Intellectual property: patents, copyright, trade marks and allied rights, 8. Aufl., London 2013, Rn. 12-57 ff. 
bei dem eine teleologische Reduktion des Ausschliesslichkeitsrechts vorgenommen wurde ${ }^{6}$. Ein anderes Beispiel ist die Tatsache, dass im Vereinigten Königreich eine Verletzungshandlung nicht bereits bei einer blossen Werkverwendung vorliegt, sondern erst dann, wenn ein wesentlicher Teil des Erstwerks übernommen wird. Damit ist der Ausschliesslichkeitsbereich bereits $a b$ initio enger umrissen. Kann das Problem aber weder auf der Ebene der Ausschliesslichkeitsrechte noch auf der Ebene der Schranken gelöst werden, ist immer noch eine Lösung ausserhalb des Urheberrechts denkbar. Viele Beispiele zeigen, dass sich Lehre und Rechtsprechung mit der Figur der impliziten Lizenz $\mathrm{zu}$ helfen wissen, auch wenn diese nach Auffassung der Autorin längerfristig nicht für einen gerechten Interessenausgleich sorgen kann.

14 Dass Probleme auf mehreren Ebenen gelöst werden können, relativiert die eindeutige Schlussfolgerung (IV.), wonach das US-Schrankensystem dank seiner fair use-Schranke am flexibelsten auf technische Veränderungen reagieren kann. Zwar schlägt die Autorin in ihren Lösungsansätzen (V.) die Einführung einer (am Ende des schweizerischen Schrankenkatalogs eingefügten) Generalklausel bzw. Öffnungsklausel vor, um zu einem offeneren System zu gelangen und so Flexibilität und Rechtssicherheit $\mathrm{zu}$ vereinen. Die Argumentation der Autorin zeigt aber, dass es Gründe gibt, diesen Weg nicht mit aller Konsequenz zu verfolgen: Die anstehenden technischen Herausforderungen weichen nicht weit von den Zwecksetzungen bestehender Schranken ab und rechtfertigen (weil Einzelanalogien möglich sind) nicht zwingend eine Öffnungsklausel; bei der Anwendung einer Generalklausel müsste der Richter die Rechtsfolgedifferenzierung vornehmen und über die Vergütung neuer Nutzungsformen entscheiden, was nicht zum Schweizer Tarifsystem passt und eben: Möglicherweise liegt die angemessene Lösung gar nicht auf der Ebene der Schranken, sondern auf jener des Ausschliesslichkeitsrechts.

So liegt der besondere Wert dieser Arbeit am Ende gerade im Verzicht auf ein flammendes Plädoyer für eine Öffnungsklausel nach Vorbild der fair useSchranke. Stattdessen zählen die leisen Differenzierungen: Das Ausleuchten der Spielräume, mögen sie noch so klein sein, das Hervorheben der Inkonsistenzen bei der Rechtsanwendung innerhalb der Rechtsordnungen und die Darstellung des Panoptikums an Möglichkeiten, um technische Neuerungen im Urheberrecht sachgerecht zu erfassen. Die beruhigende Feststellung, dass Spielräume bestehen, entbindet aber nicht von der Aufgabe, die urheberrechtlichen Schranken auch in der laufenden URG-Revision anzupassen. Ob der Gesetzgeber hingegen eine Öffnungsklausel aufnehmen sollte, lässt sich auch nach der Lektüre von Brändlis Dissertation nicht abschliessend beantworten.

«Die Flexibilität urheberrechtlicher
Schrankensysteme: Eine rechtsverglei-
chende Untersuchung am Beispiel digita-
ler Herausforderungen», Sandra Brändli,
Stämpfli Verlag AG, Bern 2017,
ISBN: 978-3-7272-1906-1

